\title{
Complete Genome Sequence Data of Three Xylella fastidiosa subsp. multiplex Strains Isolated from Olive Trees in California, U.S.A.
}

\author{
Michael L. O’Leary, ${ }^{\dagger}$ Lindsey P. Burbank, Rodrigo Krugner, and Drake C. Stenger
}

U.S. Department of Agriculture-Agricultural Research Service, San Joaquin Valley Agricultural Sciences Center, 9611 S Riverbend Ave., Parlier, CA 93648

\begin{abstract}
Xylella fastidiosa is a xylem-limited bacterial plant pathogen that causes disease on numerous hosts. Additionally, $X$. fastidiosa asymptomatically colonizes a wide range of plant species. $X$. fastidiosa subsp. multiplex has been detected in olive (Olea europaea) trees grown in California, U.S.A., as well as in Europe. Strains of $X$. fastidiosa subsp. multiplex isolated from California olive trees are not known to cause disease on olive, although some can induce leafscorch symptoms on almond (Prunus dulcis). No genome assemblies currently exist for oliveassociated $X$. fastidiosa subsp. multiplex strains; therefore, a hybrid assembly method was used to generate complete genome sequences for three $X$. fastidiosa subsp. multiplex strains (Fillmore, LM10, and RH1) isolated from olive trees grown in Ventura and Los Angeles counties of California.
\end{abstract}

\section{Resource Announcement}

Xylella fastidiosa is a xylem-limited plant pathogenic bacterium that causes disease on numerous plant hosts (Jeger et al. 2018). X. fastidiosa is divided into five genetically distinct subspecies (i.e., fastidiosa, multiplex, morus, sandyi, and pauca) that are further divided into sequence types (STs) according to a multilocus sequencing typing (MLST) classification scheme (Yuan et al. 2010). In California, U.S.A., X. fastidiosa subsp. multiplex is associated with almond (Prunus dulcis), where it causes almond leaf scorch disease, and ornamental hosts, where it may induce leaf scorch symptoms or colonize asymptomatically (Hernandez-Martinez et al. 2007). Several $X$. fastidiosa subsp. multiplex strains, e.g., Fillmore, LM10 (alias Xf10 LaMirada), $\mathrm{RH} 1$ (alias Xf11 RollingHills), and Oceanside, were isolated from different mature (i.e., at least 30 years old) ornamental olive (Olea europaea) trees with leaf scorch symptoms in Ventura, Los Angeles, and San Diego counties, respectively. However, these strains did not induce symptoms in seven olive cultivars ('Arbequina', 'Arbosana', 'Barouni', 'Koroneiki', 'Manzanillo', 'Mission', and 'Sevillano') after mechanical inoculation, and experimental infections did not persist after 1 year (Krugner et al. 2014). In contrast, three of these strains (i.e., Fillmore, LM10, and $\mathrm{RH} 1$ ) induced typical almond leaf scorch disease symptoms in mechanically inoculated almond cultivar Sonora (Krugner et al. 2014).

$X$. fastidiosa subsp. multiplex strains belonging to STs $6,7,81$, and 87 have been identified in Europe in association with a wide variety of hosts (Arias-Giraldo et al. 2020; Denance et al.

\section{${ }^{\dagger}$ Corresponding author: M. L. O’Leary; michael.oleary@usda.gov}

Mention of trade names or commercial products in this publication is solely for the purpose of providing specific information and does not imply recommendation or endorsement by the U.S. Department of Agriculture. USDA is an equal opportunity provider and employer.

The author(s) declare no conflict of interest.

Accepted for publication 11 June 2020.
Funding

Funding for this project was provided by U.S. Department of AgricultureAgricultural Research Service appropriated project \#2034-22000-01200D.

Keyword

bacteriology 
2017; Giampetruzzi et al. 2019a; Jeger et al. 2018; Landa et al. 2020; Olmo et al. 2017). Genome sequences have been assembled for several almond-infecting strains of $X$. fastidiosa subsp. multiplex isolated in Spain, including the complete genome of an ST 6 strain, IVIA5901, assembled using a hybrid method (Arias-Giraldo et al. 2020; Giampetruzzi et al. 2019b; Landa et al. 2020). In addition to causing disease on almond, STs 6 and 81 have been detected in association with olive trees in Spain, although they are not reported to cause disease on olive (Jeger et al. 2018; Landa et al. 2020); in contrast, $X$. fastidiosa subsp. pauca strains cause widespread disease in olive in Italy and have been detected in olive in Ibiza, Spain (Saponari et al. 2019). $X$. fastidiosa subsp. multiplex strains isolated in Spain and France are related to $X$. fastidiosa subsp. multiplex strains isolated from ornamental hosts in California, including LM10 and RH1, based on ST and core-genome alignment (Landa et al. 2020; Vanhove et al. 2019). To facilitate comparison of $X$. fastidiosa subsp. multiplex strains isolated in different regions, we produced high-quality complete genome sequences for $X$. fastidiosa subsp. multiplex strains Fillmore, LM10, and $\mathrm{RH} 1$, using a hybrid assembly approach.

Cultures of strains Fillmore, LM10, and RH1 were grown on PD3 agar medium at $28^{\circ} \mathrm{C}$ for 7 to 10 days; cells were subsequently collected and genomic DNA was extracted with a DNeasy Blood \& Tissue Kit (Qiagen) using the manufacturer's protocol for Gram-negative bacteria, including a 10-minute incubation with $20 \mu \mathrm{l}$ of RNase A at $20 \mathrm{mg} / \mathrm{ml}$. DNA was assessed for quality and quantity using a Nanodrop spectrophotometer (ThermoFisher Scientific), Qubit fluorometer (Invitrogen), and agarose gel imaging. The same DNA sample was used to prepare both long read and short read libraries, for sequencing on the Oxford Nanopore Technologies MinION and Illumina Novoseq platforms, respectively. For long read libraries, $1 \mu \mathrm{g}$ of DNA from strains Fillmore, LM10, and RH1 was fragmented using a g-TUBE (Covaris, Woburn, MA) following the manufacturer's instructions for 8- or 20-kbp average fragment size; for strains LM10 and RH1, an additional library was prepared with unfragmented DNA. Long read sequencing libraries were prepared with a Ligation Sequencing Kit (SQK-LSK109, Oxford Nanopore Technologies) and Native Barcoding Expansions (EXP-NDB104, Oxford Nanopore Technologies), following the manufacturer's instructions, and sequenced on a MinION device using an R9.4.1 flow cell (Oxford Nanopore Technologies). A corresponding short read library consisting of $150 \mathrm{bp}$ paired-end reads with inserts of 350 to $400 \mathrm{bp}$ was prepared by the UC Davis Genome Center DNA Technologies Core, and sequencing using the Illumina Novoseq platform, yielding 355,324,924 short read pairs for Fillmore, 328,887,528 for LM10, and $358,608,868$ for $\mathrm{RH} 1$. Nanopore long reads were base-called, filtered for quality, demultiplexed, and trimmed using Guppy v3.3.0 (Oxford Nanopore Technologies). Illumina reads were trimmed and filtered with fastp v0.20.0, then assessed for quality using fastqc v0.11.9 (http:// www.bioinformatics.babraham.ac.uk/projects/fastqc/) and multiqc v1.8 (Chen et al. 2018; Ewels et al. 2016). Analysis of all resulting fastq files with Kraken2 v2.0.8-beta using the MiniKraken2 v1 $8 \mathrm{~Gb}$ database assigned $98.6 \%$ to $99.6 \%$ of sequenced reads from each file to Xylella, verifying that contaminant (i.e., non-Xylella) sequences were not introduced during library preparation or demultiplexing steps (Wood et al. 2019). Due to the excessive short read sequencing depth (i.e., approximately 40,000x per strain), randomly subsampled sets of short read pairs, with an estimated genome coverage of 200x, were taken with Seqtk v1.3 (https:// github.com/lh3/seqtk) and used for subsequent assembly and analysis steps.

To generate complete genome assemblies, multiple combinations of assemblers, assembly parameters, and input reads were evaluated. Unicycler v0.4.8-beta was used to generate hybrid assemblies with both long and short reads, whereas Flye v2.7 was used to construct long read only assemblies (Kolmogorov et al. 2019; Wick et al. 2017). Assembly graphs were evaluated using Bandage v0.8.1 (Wick et al. 2015). For all three strains, both Unicycler and Flye failed to resolve a complete circular assembly using long reads solely from a library fragmented to $8 \mathrm{kbp}$. For Fillmore, a complete assembly was successfully generated with Flye using combined reads from 8- and 20-kbp libraries (Table 1). For strains LM10 and RH1, several combinations of assembly parameters and read sets (e.g., reads obtained from 8- and 20-kbp libraries) failed to generate complete assemblies, similar to initial failure to obtain a complete assembly of $X$. fastidiosa subsp. multiplex strain IVIA5906 encountered in a previous study, which the authors tentatively attributed to the presence of long repetitive sequences (Arias-Giraldo et al. 2020). Combining reads from the 8-kbp, 20-kbp, and unfragmented libraries successfully generated complete assemblies with Flye for strains $\mathrm{LM} 10$ and $\mathrm{RH} 1$, in conjunction with limiting the read length with Filtlong v0.2.0 (https://github.com/rrwick/Filtlong) or the "-asmcoverage' option of Flye (Table 1). For strain $\mathrm{RH} 1$, a 14.9-kbp circular contig was frequently obtained from incomplete assemblies; however, long read alignment with minimap2 v2.16 
TABLE 1. Summary of methods used and characteristics of genome assemblies of Fillmore, LM10, and RH1, and comparison with Xylella fastidiosa subsp. multiplex strain M12

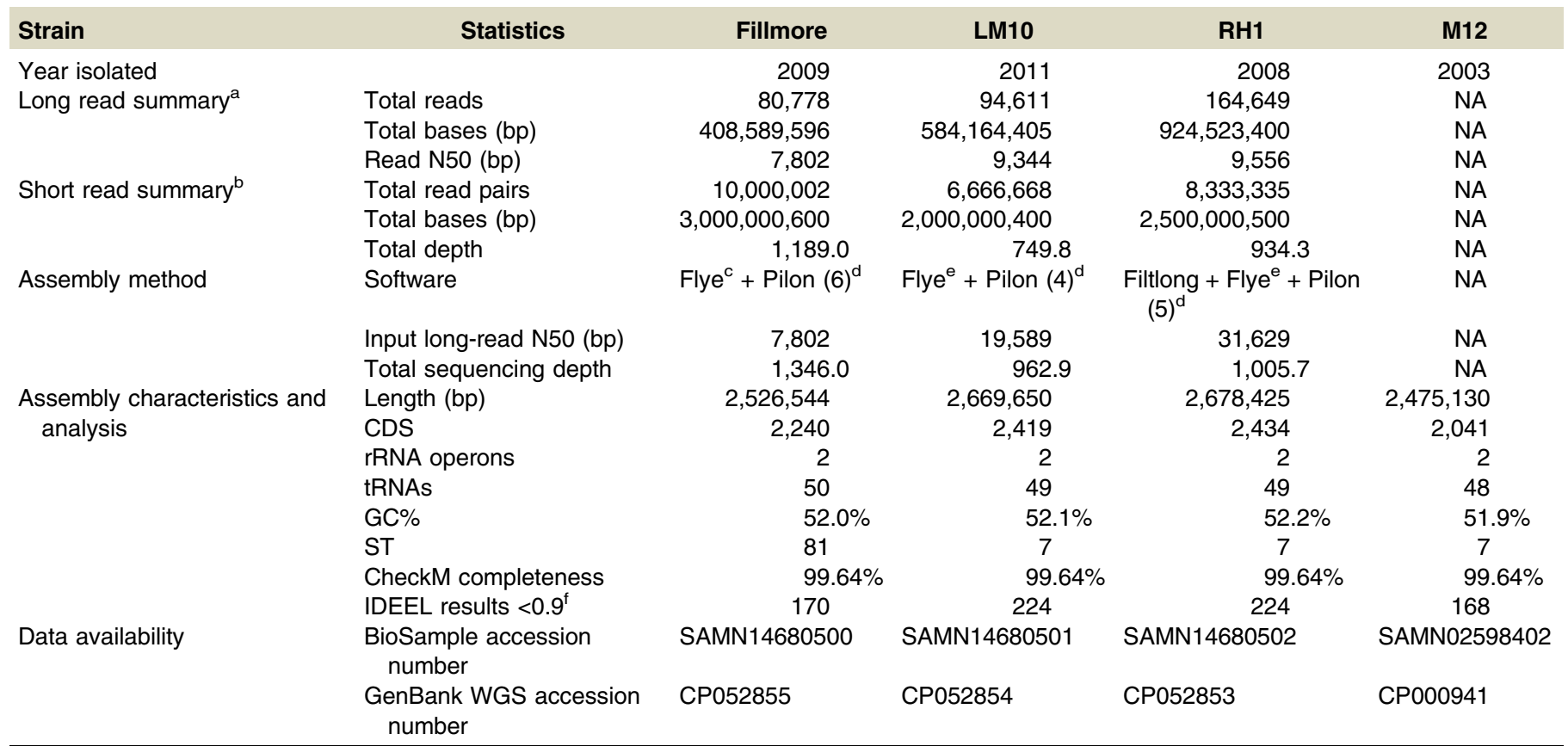

a Combined values for all fragment size libraries used per strain, e.g., $8 \mathrm{kbp}, 20 \mathrm{kbp}$, unfragmented.

b Summary of all randomly subsampled reads used.

c Run with '-plasmids' option.

d Indicates the number of times Pilon was run.

e Run with '-plasmids' and '-asm-coverage 80' options.

$f$ Length ratio of each protein coding sequence to the best match in UniProt TREMBL. A ratio $<0.9$ suggests a protein coding sequence is smaller than expected and may be erroneously disrupted.

supports the inclusion of this sequence on the chromosome as a 31-kbp region (positions 1505426 to 1536384 ) containing two overlapping 16.1-kbp repeats (Li 2018). Once complete assemblies were obtained, each chromosome was rotated to begin at the first nucleotide position of the dnaA gene, and the assembly polished with Pilon multiple times to correct insertion/deletion errors common in long read assemblies that can interfere with accurate open reading frame prediction and downstream analysis (Walker et al. 2014; Watson and Warr 2019). Each polishing iteration used a new random subsample of Illumina read pairs, generated with Seqtk.

Polished assemblies were evaluated for completeness with CheckM v1.1.2, and for disrupted protein coding sequences with IDEEL (Parks et al. 2015; Stewart et al. 2019). As a control, both analyses included the complete genome assembly of $X$. fastidiosa subsp. multiplex ST 7 strain M12 (GenBank accession CP000941.1), isolated from almond in 2003 and assembled with data generated from Sanger sequencing (Chen et al. 2010). CheckM estimates the genome assemblies of Fillmore, LM10, and RH1 to be $99.64 \%$ complete, and assigns an identical score to M12. IDEEL identified 170 to 224 potentially truncated protein coding sequences (i.e., ratio of coding sequence length to that of the best match in UniProt TREMBL database <0.9) in Fillmore, LM10, and RH1, whereas IDEEL identified 168 in M12 (Table 1). Polished assemblies were annotated using the NCBI Prokaryotic Genome Annotation Pipeline (Tatusova et al. 2016).

A summary of assembly methods used and features for the polished genome assembly of each strain is shown in Table 1. No plasmids were identified in these strains. MLST analysis of housekeeping genes utilizing the $X$. fastidiosa PubMLST database (https://pubmlst.org/ xfastidiosa/) identified LM10 and RH1 as ST 7, whereas Fillmore is ST 81. Strains of ST 7 and ST 81 have a similar MLST allelic profile, differing at only at CysG (allele 7 and allele 32, respectively). $X$. fastidiosa subsp. multiplex strains belonging to ST 7 and ST 81 have been previously identified in Europe, and draft genomes exist for a small number of European ST 7 (e.g., CFBP8416) and ST 81 (e.g., XF3348, XYL1752, and XYL1981) strains (Denance et al. 2017; Landa et al. 2020). Strains belonging to ST 81 have been detected in association with, or 
isolated from, numerous hosts in the Balearic Islands of Spain, including almond, fig (Ficus carica), olive, plum (Prunus domestica), and several landscape or ornamental hosts (Jeger et al. 2018; Landa et al. 2020). The genome assembly of $X$. fastidiosa subsp. multiplex strain Fillmore represents the first genome sequence of an ST 81 strain originating from North America. Several recent studies have suggested whole-genome sequencing data for $X$. fastidiosa are informative for characterizing of strains involved in outbreaks, as the resolution provided by MLST analysis may be limited (Arias-Giraldo et al. 2020; Landa et al. 2020); comparison between Fillmore and Spanish ST 81 strains may provide additional insight into the origins of those strains.

Data availability. Data for the genome assemblies of $X$. fastidiosa subsp. multiplex strains Fillmore, LM10, and RH1 are associated with BioProject PRJNA627683 and BioSamples SAMN14680500 to SAMN14680502. Sequencing reads used to generate assemblies were deposited into the Sequence Read Archive (SRA) under accession numbers SRR11638828 to SRR11638838. Genome assemblies have been deposited into GenBank under accession numbers CP052853 to CP052855.

\section{Acknowledgments}

We thank Kunbo Zhang and Brandon Ortega for technical support. Illumina sequencing was done by the UC Davis Genome Center DNA Technologies and Expression Analysis Cores, supported by NIH Shared Instrumentation Grant 1S10OD010786-01.

\section{Literature Cited}

Arias-Giraldo, L. F., Giampetruzzi, A., Metsis, M., Marco-Noales, E., Imperial, J., Velasco-Amo, M. P., Roman-Ecija, M., and Landa, B. B. 2020. Complete circularized genome data of two Spanish strains of Xylella fastidiosa (IVIA5235 and IVIA5901) using hybrid assembly approaches. Phytopathology 110:969-972.

Chen, J., Xie, G., Han, S., Chertkov, O., Sims, D., and Civerolo, E. L. 2010. Whole genome sequences of two Xylella fastidiosa strains (M12 and M23) causing almond leaf scorch disease in California. J. Bacteriol. 192:4534.

Chen, S., Zhou, Y., Chen, Y., and Gu, J. 2018. fastp: An ultra-fast all-in-one FASTQ preprocessor. Bioinformatics 34:1884-i890.

Denance, N., Legendre, B., Briand, M., Olivier, V., de Boisseson, C., Poliakoff, F., and Jacques, M. A. 2017. Several subspecies and sequence types are associated with the emergence of Xylella fastidiosa in natural settings in France. Plant Pathol. 66: 1054-1064.

Ewels, P., Magnusson, M., Lundin, S., and Kaller, M. 2016. MultiQC: Summarize analysis results for multiple tools and samples in a single report. Bioinformatics 32 : 3047-3048.

Giampetruzzi, A., D’Attoma, G., Zicca, S., Abou Kubaa, R., Rizzo, D., Boscia, D., Saldarelli, P., and Saponari, M. 2019a. Draft genome sequence resources of three strains (TOS4, TOS5, and TOS14) of Xylella fastidiosa infecting different host plants in the newly discovered outbreak in Tuscany, Italy. Phytopathology 109:1516-1518.

Giampetruzzi, A., Velasco-Amo, M. P., Marco-Noales, E., Montes-Borrego, M., RomanEcija, M., Navarro, I., Monterde, A., Barbe, S., Almeida, R. P. P., Saldarelli, P., Saponari, M., Montilon, V., Savino, V. N., Boscia, D., and Landa, B. B. 2019b. Draft genome resources of two strains ("ESVL" and "IVIA5901") of Xylella fastidiosa associated with almond leaf scorch disease in Alicante, Spain. Phytopathology 109: 219-221.

Hernandez-Martinez, R., de la Cerda, K. A., Costa, H. S., Cooksey, D. A., and Wong, F. P. 2007. Phylogenetic relationships of Xylella fastidiosa strains isolated from landscape ornamentals in southern California. Phytopathology 97:857-864.

Jeger, M., Caffier, D., Candresse, T., Chatzivassiliou, E., Dehnen-Schmutz, K., Gilioli, G., Gregoire, J. C., Miret, J. A. J., MacLeod, A., Navarro, M. N., Niere, B., Parnell, S., Potting, R., Rafoss, T., Rossi, V., Urek, G., Van Bruggen, A., Van der Werf, W., West, J., Winter, S., Almeida, R., Bosco, D., Jacques, M. A., Landa, B., Purcell, A., Saponari, M., Czwienczek, E., Delbianco, A., Stancanelli, G., and Bragard, C. 2018. Updated pest categorisation of Xylella fastidiosa. EFSA J. 16:5357.

Kolmogorov, M., Yuan, J., Lin, Y., and Pevzner, P. A. 2019. Assembly of long, errorprone reads using repeat graphs. Nat. Biotechnol. 37:540-546.

Krugner, R., Sisterson, M. S., Chen, J. C., Stenger, D. C., and Johnson, M. W. 2014. Evaluation of olive as a host of Xylella fastidiosa and associated sharpshooter vectors. Plant Dis. 98:1186-1193.
Landa, B. B., Castillo, A. I., Giampetruzzi, A., Kahn, A., Roman-Ecija, M., Velasco-Amo, M. P., Navas-Cortes, J. A., Marco-Noales, E., Barbe, S., Moralejo, E., Coletta, H. D., Saldarelli, P., Saponari, M., and Almeida, R. P. P. 2020. Emergence of a plant pathogen in Europe associated with multiple intercontinental introductions. Appl. Environ. Microbiol. 86.

Li, H. 2018. Minimap2: Pairwise alignment for nucleotide sequences. Bioinformatics 34:3094-3100

Olmo, D., Nieto, A., Adrover, F., Urbano, A., Beidas, O., Juan, A., Marco-Noales, E., Lopez, M. M., Navarro, I., Monterde, A., Montes-Borrego, M., Navas-Cortes, J. A., and Landa, B. B. 2017. First detection of Xylella fastidiosa infecting cherry (Prunus avium) and Polygala myrtifolia plants, in Mallorca Island, Spain. Plant Dis. 101:1820.

Parks, D. H., Imelfort, M., Skennerton, C. T., Hugenholtz, P., and Tyson, G. W. 2015. CheckM: Assessing the quality of microbial genomes recovered from isolates, single cells, and metagenomes. Genome Res. 25:1043-1055.

Saponari, M., Giampetruzzi, A., Loconsole, G., Boscia, D., and Saldarelli, P. 2019. Xylella fastidiosa in olive in Apulia: Where we stand. Phytopathology 109:175-186.

Stewart, R. D., Auffret, M. D., Warr, A., Walker, A. W., Roehe, R., and Watson, M. 2019. Compendium of 4,941 rumen metagenome-assembled genomes for rumen microbiome biology and enzyme discovery. Nat. Biotechnol. 37:953-961.

Tatusova, T., DiCuccio, M., Badretdin, A., Chetvernin, V., Nawrocki, E. P., Zaslavsky, L., Lomsadze, A., Pruitt, K., Borodovsky, M., and Ostell, J. 2016. NCBI prokaryotic genome annotation pipeline. Nucleic Acids Res. 44:6614-6624.

Vanhove, M., Retchless, A. C., Sicard, A., Rieux, A., Coletta, H. D., De La Fuente, L., Stenger, D. C., and Almeida, R. P. P. 2019. Genomic diversity and recombination among Xylella fastidiosa subspecies. Appl. Environ. Microbiol. 85.

Walker, B. J., Abeel, T., Shea, T., Priest, M., Abouelliel, A., Sakthikumar, S., Cuomo, C. A., Zeng, Q. D., Wortman, J., Young, S. K., and Earl, A. M. 2014. Pilon: An integrated tool for comprehensive microbial variant detection and genome assembly improvement. PLoS One 9:e112963.

Watson, M., and Warr, A. 2019. Errors in long-read assemblies can critically affect protein prediction. Nat. Biotechnol. 37:124-126.

Wick, R. R., Judd, L. M., Gorrie, C. L., and Holt, K. E. 2017. Unicycler: Resolving bacterial genome assemblies from short and long sequencing reads. PLOS Comput. Biol. 13:e1005595.

Wick, R. R., Schultz, M. B., Zobel, J., and Holt, K. E. 2015. Bandage: Interactive visualization of de novo genome assemblies. Bioinformatics 31:3350-3352.

Wood, D. E., Lu, J., and Langmead, B. 2019. Improved metagenomic analysis with Kraken 2. Genome Biol. 20:257.

Yuan, X., Morano, L., Bromley, R., Spring-Pearson, S., Stouthamer, R., and Nunney, L. 2010. Multilocus sequence typing of Xylella fastidiosa causing Pierce's disease and oleander leaf scorch in the United States. Phytopathology 100:601-611. 